\title{
ANÁLISE COMPARATIVA DAS EMISSÕES OTOACÚSTICAS COM A TIMPANOMETRIA EM LACTENTES DE 0 A 6 MESES
}

\author{
Comparative analysis of otoacoustic emissions \\ with tympanometry in 0-6 month infants
}

\author{
Elizângela Dias Camboim (1), Aline Mabel Novaes Correia (2), Dênis Vasconcelos ${ }^{(3)}$, \\ Raquel Torres ${ }^{(4)}$, Renata Coelho Scharlach ${ }^{(5)}$, Marisa Frasson de Azevedo ${ }^{(6)}$
}

\section{RESUMO}

Objetivo: comparar os achados das emissões otoacústicas evocadas por estímulos transientes (EOAT) com a timpanometria usando tom sonda de 226 e $1000 \mathrm{~Hz}$ em lactentes. Método: o estudo foi realizado no Serviço de Otorrinolaringologia do Hospital Santa Juliana de Maceió - Alagoas. Selecionaram-se lactentes de ambos os gêneros, com faixa etária de recém nascido a seis meses. Foram excluídos aqueles com fissura lábio palatinos, malformação de orelha externa e/ou média, síndromes e história familiar de perda auditiva. Foram realizados os seguintes procedimentos: anamnese para identificar as queixas auditivas e selecionar a amostra de acordo com os critérios de inclusão e exclusão; avaliação otorrinolaringológica por meio de otoscopia e avaliação auditiva que se constituiu de emissões otoacústicas evocadas por estímulo transiente (EOAET) e timpanometria com tom teste da sonda de 226 e $1000 \mathrm{~Hz}$. Para analisar as variáveis qualitativas, foi utilizado o teste de Igualdade de Duas Proporções, além do teste Qui-Quadrado para Independência e o intervalo de confiança para a Média, utilizados para a análise das variáveis quantitativas dos resultados. Resultados: comparando-se os resultados das EOAT com os resultados da timpanometria obtida com os tons testes de $226 \mathrm{~Hz}$ e $1000 \mathrm{~Hz}$, observou-se uma associação da EOAT com a sonda de $1000 \mathrm{~Hz}$ em ambas as orelhas, ou seja, houve ausência das EOAT nos lactentes que apresentaram timpanogramas alterados e presença quando os timpanogramas estavam normais. Conclusão: a timpanometria com sonda de $1000 \mathrm{~Hz}$ teve maior correlação com as EOAT tanto para identificação de respostas em orelhas normais como para as alterações de orelha média.

DESCRITORES: Emissões Otoacústicas Espontâneas; Testes de Impedância Acústica; Otoscopia

(1) Fonoaudióloga; Professora auxiliar da disciplina de Abordagem Terapêutica em Audiologia da Faculdade de Fonoaudiologia de Alagoas - UNCISAL; Especialista em Audiologia Clínica pela Universidade Federal de Pernambuco.

(2) Graduanda de Fonoaudiologia da Universidade Estadual de Ciências da Saúde de Alagoas - UNCISAL.

(3) Médico otorrinolaringologista do Instituto do Otorrinolaringologia do Hospital Santa Juliana.

(4) Fonoaudióloga do Instituto do Otorrinolaringologia do Hospital Santa Juliana; Especialista em Audiologia.

(5) Fonoaudióloga; Professora do Programa de Mestrado Profissional em Reabilitação do Equilíbrio Corporal e Inclusão Social da Universidade Bandeirante de São Paulo; Professora colaboradora da Disciplina de Distúrbios da Audição do Departamento de Fonoaudiologia da Universidade Federal de São Paulo e fonoaudióloga do Núcleo de Estudos Fonoaudiológicos; Doutora em Distúrbios da Comunicação Humana (Fonoaudiologia) pela Universidade Federal de São Paulo.

\section{INTRODUÇÃO}

O diagnóstico precoce da perda auditiva em neonatos constitui-se em estratégia fundamental para o planejamento e introdução de medidas terapêuticas, objetivando a prevenção de agravos e melhoria da qualidade de vida. Testes objetivos, como as emissões otoacústicas, têm maior especificidade e sensibilidade para detectar estas possíveis alterações, principalmente em recém-nascidos e crianças pequenas ${ }^{1,2}$.

(6) Fonoaudióloga; Professor Associado Doutor da Universidade Federal de São Paulo; Doutora em Distúrbios da Comunicação Humana pela Universidade Federal de São Paulo.

Conflito de interesses: inexistente 
O registro das Emissões Otoacústicas tem sido amplamente indicado, por ser um exame rápido, de fácil aplicação e permite testar grande número de sujeitos em curto espaço de tempo, sendo, portanto, útil nos programas de triagem auditiva neonatal ${ }^{3,4}$.

Apesar de não quantificarem a deficiência auditiva, as emissões otoacústicas permitem a detecção da lesão localizada no órgão de Corti por meio da avaliação da função de células ciliadas externas (CCE). Este tipo de exame é extremamente sensível às alterações de orelha média $(\mathrm{OM})$, uma vez que não é possível captar a energia sonora vinda da cóclea após estimulação sonora gerada pelo otoemissor ${ }^{5}$.

O principal método de avaliação da orelha média é a imitanciometria, que por meio de uma medida dinâmica (timpanometria) avalia a mobilidade tímpano-ossicular, além da pesquisa do reflexo acústico. Essas medidas se tornam importantes devido à alta incidência de problemas de orelha média nos recém-nascidos. O uso combinado de timpanometria e pesquisa do reflexo acústico acrescenta precisão no diagnóstico de alteração de $\mathrm{OM}^{6}$.

O acompanhamento auditivo periódico para lactentes nos dois primeiros anos de vida é de extrema importância, considerando ser este o período crítico para o processo de maturação do sistema auditivo, no qual privações sensoriais auditivas podem ser responsáveis por sequelas para o desenvolvimento de fala e linguagem ${ }^{7,1}$.

De acordo com a literatura, a maioria dos recém-nascidos com efusão de orelha média neonatal persistente desenvolve otite média crônica com efusão durante o primeiro ano de vida, com presença de um ou mais episódios de secreção na orelha. Quanto mais cedo ocorrer o primeiro episódio, maior a probabilidade de recorrência, sendo que o período de aleitamento materno exclusivo parece atuar como um fator protetor ${ }^{8}$.

Pesquisas demonstram que a perda auditiva condutiva é um achado muito frequente nessas populações, havendo correlação significante entre falha na triagem auditiva e os riscos: antecedente familiar e síndrome, como também, com a idade gestacional e o peso ao nascimento ${ }^{9-10}$.

A timpanometria pode ser realizada com sonda de mutifrequências. A sonda de $226 \mathrm{~Hz}$ é muito utilizada na prática clínica, a qual é bastante eficiente na identificação de alterações de orelha média nas crianças acima de 6 meses. No entanto, pesquisas mostram que no lactente abaixo de 6 meses, este tipo de tom não tem se mostrado muito eficaz, pois é possível encontrar resultados timpanométricos normais (tipo A) mesmo na presença de comprometimento de orelha média. A utilização da sonda com tom de frequência mais alta, como 660 e $1000 \mathrm{~Hz}$, em neonatos tem sido considerada mais eficiente $e$ sensível na identificação de alterações do sistema tímpano-ossicular em crianças com idade inferior a 6 meses, sendo importante a realização deste exame dentro de um programa de TAN ${ }^{11-13}$.

Desta forma, se fez necessária a realização desta pesquisa com o objetivo de comparar os achados das emissões otoacústicas evocadas por estímulos transientes (EOAT) com a timpanometria usando tom sonda de 226 e $1000 \mathrm{~Hz}$ em lactentes.

\section{MÉTODO}

Estudo transversal e de correlação de dois exames, com mascaramento dos avaliadores, ou seja, duplo cego, pois cada avaliação foi realizada separadamente, por diferentes avaliadores no mesmo dia, sem que os examinadores tivessem conhecimento dos resultados das demais avaliações, antes da finalização de todos os testes. O estudo foi aprovado pelo Comitê de Ética em Pesquisa da Universidade Estadual de Ciências da Saúde de Alagoas - UNCISAL, mediante o parecer $\mathrm{n}^{\circ}$ 829. Antes do início do teste o termo de consentimento livre e esclarecido foi lido e explicado aos responsáveis pelos bebês, os quais concordaram e assinaram.

Foram avaliados 118 lactentes de ambos os sexos, com faixa etária de $\mathrm{RN}$ a seis meses, nascidos pré termo ou a termo encaminhados por pediatras da rede pública e particular da cidade.

Foram excluídos aqueles que apresentavam fissura lábio palatino ou malformação de orelha externa e ou média, malformação de cabeça e pescoço, síndromes genéticas associadas a alterações auditivas, crianças com história familiar de perda auditiva e alterações neurológicas.

O estudo foi realizado no Serviço de Otorrinolaringologia do Hospital Santa Juliana (Instituição Filantrópica Municipal), Maceió - Alagoas.

Inicialmente foi realizada a anamnese (Figura 1) com a mãe ou responsável, para identificar riscos que poderiam causar a alteração auditiva e selecionar a amostra de acordo com os critérios de inclusão e exclusão. Posteriormente, foram realizados os seguintes procedimentos: otoscopia, emissões otoacústicas evocadas por estímulos transientes e timpanometria com tom sonda de 226 e $1000 \mathrm{~Hz}$. A ordem de realização dos procedimentos foram aleatórias e acontecia de acordo com a disponibilidade do profissional.

A otoscopia foi realizada por um médico otorrinolaringologista, com otoscópio Hinne de marca Welch allyn para inspeção da orelha externa e média, a fim de identificação de rolha de cerume 
Nome:

$\mathrm{D} / \mathrm{N}:$ Idade: Encaminhado por:

Nome da Mãe: Data: Idade:

Endereço:

Telefone:

IG: $\mathrm{P} / \mathrm{N}$ :

$P A:$

Apgar: Tipo de parto: Capurro somático:

Intercorrências Gestacionais:

HIPERTENSÃO ( ) NEFROPATIA ( ) ALTERAÇÃO ENDÓCRINA ( ) T ( ) PT ( )

\section{Intercorrências neonatais:}

HIPÓXIA ( ) ICTERÍCIA ( ) Outros:

Indicadores de Risco para Perda Auditiva:

( ) Antecedentes Familiares

( ) Consangüinidade

( ) Infecções Congênitas

( ) Mal formação Crânio Facial

( ) Peso < 1.500g ou PIG

( ) Hiperbilirrubinemia

( ) Ototóxico Qual:

( ) Meningite Bacteriana

( ) Apgar 0/4 no $1^{\circ}$ minuto e 0/6 no $5^{\circ}$ minuto

( ) Ventilação mecânica (> 5dias)

( ) Síndromes

( ) Alcoolismo materno ou uso de drogas

( ) Hemorragia ventricular

( ) Incubadora (> 7 dias)

( ) Convulsões neonatais

( ) Otite média recorrente/ persistente (> 3 meses)

( ) Suspeita de atraso do desenvolvimento de linguagem, audição e fala.

Refluxo: Fisiológico（） Patológico（） sem RGE（）

Figura 1 - Anamnese 
no meato acústico externo ou presença de corpo estranho, que impedissem a realização do exame.

A avaliação da função auditiva periférica foi realizada pela pesquisadora responsável, por meio dos exames de emissões otoacústicas evocadas por estímulo transiente com cliques não lineares na intensidade de 83 dBNPS, utilizando-se o equipamento de marca Interacoustic modelo OtoRead.

Os parâmetros "PASSA /FALHA" utilizados para esta pesquisa foram os descritos no protocolo do próprio equipamento: estímulo tipo clique; intensidade de $83 \mathrm{dBSPL}$; número de bandas de frequências testadas: seis (de $1500 \mathrm{~Hz}$ a $4000 \mathrm{~Hz}$ ). Foi considerado o resultado "PASSA" quando as emissões se apresentavam numa relação sinal/ruído de $4 \mathrm{~dB}$ em pelo menos três bandas de frequência.

As medidas de Imitância Acústica foram realizadas antes ou depois das emissões, por uma segunda avaliadora (Fonoaudióloga), sendo utilizado o equipamento da Interacoustic modelo AT $235 \mathrm{~h}$. Foi realizada a timpanometria com tom de sonda de 226 e $1000 \mathrm{~Hz}$ sendo aleatória a escolha da frequência para iniciar o teste em cada lactente, bem como o lado da orelha.

Os tipos de timpanogramas foram classificados segundo Jerger (1970); Margolis (2003) em:

- Curva Tipo A - pico único de admitância entre -150 e + $100 \mathrm{daPa}$

- Curva Tipo C - pico de admitância deslocado para pressão negativa

- Curva tipo D - curva em duplo pico, considerado normal em RN

- Curva tipo Ad - pico de máxima complacência com amplitude aumentada.

- Curva tipo Ar - pico de máxima complacência com amplitude reduzida.

- Curva tipo B curva plana sem pico de adimitância.

Para classificação dos timpanogramas "Ad" e "Ar" além do critério de Jerger (1970), foram utilizadas a normatização do volume da admitância proposta pela ASHA (American Speech-LanguageHearing Association) 1999, que padronizou valores normais de 0,3 a $1,4 \mathrm{ml}$. Com isso foram considerados no presente estudo, valores abaixo de $0,3 \mathrm{ml}$ como timpanograma do tipo "Ar" e acima de $1,4 \mathrm{ml}$ timpanograma "Ad".

Para análise dos resultados utilizou-se um critério simplificado de classificação dos timpanogramas: normais ou alterados. Sendo considerado normais todos os timpanogramas do tipo "A" e "duplo pico" e alterados os demais.

Ao término de todos os exames, os testes foram impressos e entregues aos responsáveis do lactente. As crianças que confirmaram a alteração foram encaminhadas para o serviço de otorrinolaringologia na Faculdade de Fonoaudiologia da UNCISAL para tratamento completo.

Os dados obtidos, incluindo os resultados dos testes de avaliação auditiva e avaliação otorrinolaringológica, foram anotados em um formulário padronizado. (Figura 2).

O estudo foi aprovado pelo Comitê de Ética em Pesquisa da Universidade Estadual de Ciências da Saúde de Alagoas - UNCISAL, mediante o parecer $\mathrm{n}^{\circ} 829$. Antes do início do teste o termo de consentimento livre e esclarecido foi lido e explicado aos responsáveis pelos bebês, os quais concordaram e assinaram.

Para analisar as variáveis qualitativas (distribuição dos lactentes em relação à variável gênero $e$ idade gestacional, bem como comparação das EOA e timpanometria em relação também à variável gênero) foi utilizado o teste de Igualdade de Duas

Proporções; O teste Qui-Quadrado para independência foi utilizado na análise das variáveis quantitativas, para medir a associação dos resultados das EOAT com a timpanometria nas sondas de 226 e $1000 \mathrm{~Hz}$. Sendo considerado o nível de significância quando o $p$-valor foi menor que 0,05 (5\%) e com intervalos de confiança de $95 \%$. Todos os valores significantes estão marcados com $\left(^{*}\right)$.

\section{RESULTADOS}

$\mathrm{Na}$ avaliação da distribuição da variável gênero, foi encontrado no presente estudo um maior percentual de lactentes do gênero feminino, que é apresentado na Tabela 1, a qual caracteriza a amostra na distribuição dos lactentes em relação à variável gênero.

A distribuição dos lactentes em relação à variável idade gestacional (nascidos a termo e pré termo) é apresentado na Tabela 2, sendo encontrada maior ocorrência de lactentes nascidos a termo.

$\mathrm{Na}$ associação das EOA com a timpanometria foi utilizado o teste de Qui-Quadrado para Independência, a fim de medir o grau de relação entre EOA (amplitude geral) com a classificação de timpanometria. As curvas timpanométricas foram classificadas em: normal (tipo A e duplo pico) e alterado (demais tipos). Essa relação e/ou associação foi medida por orelha.

Os resultados de associação de qui-quadrado são apresentados em valores absolutos e percentuais. Nas Tabelas 3 e 4, é possível observar a distribuição conjunta dos resultados das EOA e timpanometria e suas combinações, tanto para a orelha direita como para orelha esquerda. 


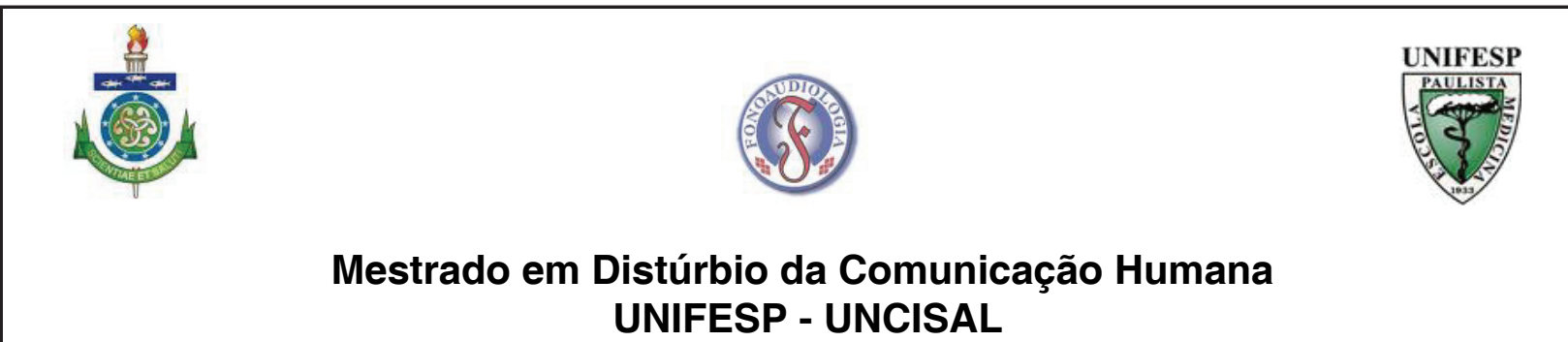

\section{Folha de Resultado}

Emissões Otoacústicas por Estímulo Transiente:

OD:

$\mathrm{OE}:$

Imitanciometria:

Avaliação ORL

( ) Opaca

( ) Descamação

( ) Hiperemiada

( ) Abaulada

( ) Retraída

( ) Perfurada

( ) Com efusão

( ) Normal

Figura 2 - Formulário padronizado de coleta otorrinolaringológica e resultado

Ao se comparar a distribuição da coluna de total com a distribuição das demais colunas (intermediárias), definiu-se que houve uma associação e/ou dependência estatística quando algum valor por linha esteve muito diferente do valor da coluna total.

Nesta análise foi possível observar que houve uma maior associação das EOAT com o timpanograma na sonda de $1000 \mathrm{~Hz}$ em lactentes para ambos os lados. Nas orelhas direitas houve concordância entre os resultados alterados (ausência de EOA com alteração na Timpanometria) em $73 \%$ e entre os resultados normais (presença de EOA e timpanometria normal) a concordância foi de (71\%). Nas orelhas esquerdas a concordância entre as resultados alterados foi de $62 \%$ e entre os normais foi de $70 \%$ (tabelas 3 e 4).

Por fim, foi realizada comparação entre os gêneros na distribuição da frequência relativa (percentuais) de todas as variáveis qualitativas, utilizando o teste de Igualdade de Duas Proporções (Tabela 5). Não sendo observado diferença estatística entre os dois gêneros quanto à distribuição dos resultados de EOA e Timpanometria, apesar de ser evidenciada diferença entre os grupos. 
Tabela 1 - Distribuição dos lactentes em relação à variável gênero

\begin{tabular}{lcc}
\hline & $\mathbf{N}$ & $\%$ \\
\hline Fem & 66 & $55,93 \%$ \\
Masc & 52 & $44,07 \%$ \\
TOTAL & 118 & $100,00 \%$ \\
\hline
\end{tabular}

Tabela 2 - Distribuição dos lactentes em relação à variável idade gestacional

\begin{tabular}{lcc}
\hline & $\mathbf{N}$ & $\%$ \\
\hline Pré-Termo & 34 & $28,81 \%$ \\
Termo & 84 & $71,19 \%$ \\
TOTAL & 118 & $100,00 \%$ \\
\hline
\end{tabular}

Legenda: IG: idade gestacional.

Tabela 3 - Associação dos Resultados das EOAT com o resultado da Timpanometria com sonda de $226 \mathrm{~Hz}$ e de $1000 \mathrm{~Hz}$ para a OD

\begin{tabular}{ccccccccc}
\hline \multirow{2}{*}{ OD } & \multirow{2}{*}{ Ausente } & \multicolumn{2}{c}{ Presente } & \multicolumn{3}{c}{ Total } & \multirow{2}{*}{ p-valor } \\
\cline { 3 - 7 } & & $\mathbf{N}$ & $\%$ & $\mathbf{N}$ & $\%$ & $\mathbf{N}$ & $\%$ & \\
\hline \multirow{2}{*}{ Timp } & Alterado & 4 & $36 \%$ & 32 & $30 \%$ & 36 & $31 \%$ & \\
$226 \mathrm{~Hz}$ & Normal & 7 & $64 \%$ & 75 & $70 \%$ & 82 & $69 \%$ & 0,658 \\
& TOTAL & 11 & $100 \%$ & 107 & $100 \%$ & 118 & $100 \%$ & \\
\hline \multirow{2}{*}{ Timp 1 } & Alterado & 8 & $73 \%$ & 31 & $29 \%$ & 39 & $33 \%$ & \\
kHz & Normal & 3 & $27 \%$ & 76 & $71 \%$ & 79 & $67 \%$ & $0,003^{*}$ \\
& TOTAL & 11 & $100 \%$ & 107 & $100 \%$ & 118 & $100 \%$ & \\
\hline
\end{tabular}

${ }^{*} \mathrm{p}$-valor< 0,005. Legenda: OD: orelha direita; Timp $226 \mathrm{~Hz}$ : timpanometria com sonda de $226 \mathrm{~Hz}$; Timp. $1000 \mathrm{~Hz}$ : timpanometria com sonda de $1000 \mathrm{~Hz}$.

Tabela 4 - Associação dos Resultados das EOAT com o resultado da Timpanometria com sonda de $226 \mathrm{~Hz}$ e de $1000 \mathrm{~Hz}$ para a OE

\begin{tabular}{|c|c|c|c|c|c|c|c|c|}
\hline \multirow{2}{*}{\multicolumn{2}{|c|}{ OE }} & \multicolumn{2}{|c|}{ Ausente } & \multicolumn{2}{|c|}{ Presente } & \multicolumn{2}{|c|}{ Total } & \multirow{2}{*}{ p-valor } \\
\hline & & $\mathbf{N}$ & $\%$ & $\mathbf{N}$ & $\%$ & $\mathbf{N}$ & $\%$ & \\
\hline \multirow{3}{*}{$\begin{array}{c}\text { Timp } \\
226 \mathrm{~Hz}\end{array}$} & Alterado & 6 & $46 \%$ & 28 & $27 \%$ & 34 & $29 \%$ & \multirow{3}{*}{0,143} \\
\hline & Normal & 7 & $54 \%$ & 77 & $73 \%$ & 84 & $71 \%$ & \\
\hline & TOTAL & 13 & $100 \%$ & 105 & $100 \%$ & 118 & $100 \%$ & \\
\hline \multirow{3}{*}{$\begin{array}{c}\text { Timp } 1 \\
\text { kHz }\end{array}$} & Alterado & 8 & $62 \%$ & 31 & $30 \%$ & 39 & $33 \%$ & \multirow{3}{*}{$0,021^{*}$} \\
\hline & Normal & 5 & $38 \%$ & 74 & $70 \%$ & 79 & $67 \%$ & \\
\hline & TOTAL & 13 & $100 \%$ & 105 & $100 \%$ & 118 & $100 \%$ & \\
\hline
\end{tabular}

*p-valor<0,005. Legenda: OE: orelha esquerda; Timp $226 \mathrm{~Hz}$ : timpanometria com sonda de $226 \mathrm{~Hz}$; Timp. $1000 \mathrm{~Hz}$ : timpanometria com sonda de $1000 \mathrm{~Hz}$. 
Tabela 5 - Comparação das EOA e resultados da timpanometria em relação à variável gênero

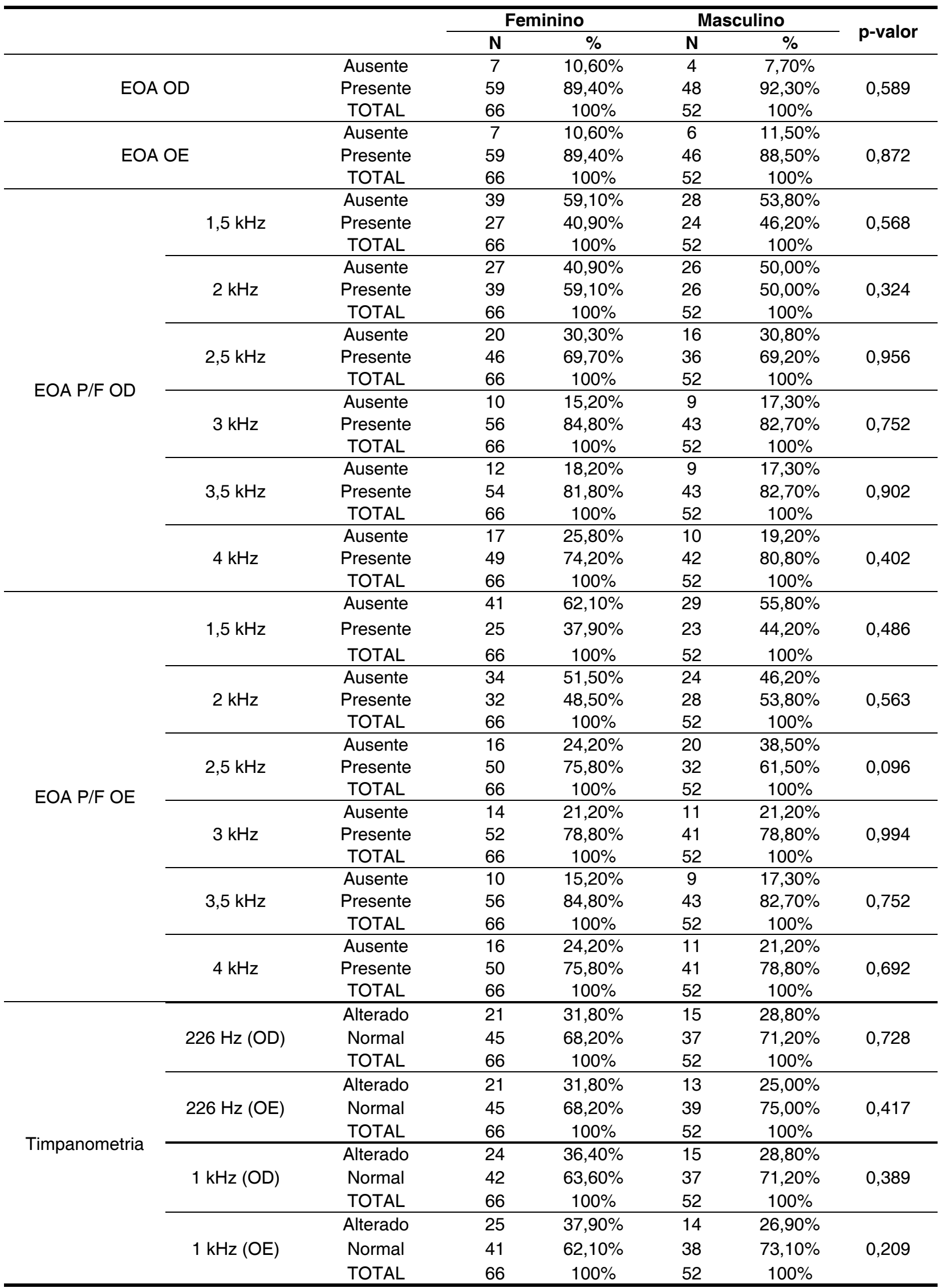

Legenda: EOA: emissões otoacústicas;P/F: passou/ falhou; OD: Orelha direita; OE: orelha esquerda. 


\section{DISCUSSÃO}

No presente estudo constatou-se predomínio de lactentes nascidos a termo e do sexo feminino (Tabelas 1 e 2 ).

Comparando-se as EOAT com uso de diferentes tons de sonda (Tabelas 3 e 4), observou-se uma associação da EOAT com a sonda de $1000 \mathrm{~Hz}$ em ambas as orelhas, pois a maioria dos lactentes com ausência das EOA apresentaram timpanogramas alterados e a maioria dos lactentes com EOA presentes apresentaram timpanogramas normais. A importância da correlação e/ou associação entre os resultados da timpanometria e emissões otoacústicas já havia sido relatada por alguns autores que encontraram correlações significantes das EOA com uso da sonda de $1000 \mathrm{~Hz}$ em lactentes de 0 a 4 meses. Esses achados estão de acordo com outros estudos que observaram uma maior adimitância com sonda de $1000 \mathrm{~Hz}$ em bebês que passaram nas EOA e encontraram associação das falhas das EOA com a alteração na timpanometria, sendo indicativo de função normal de orelha média quando as EOA estão presentes associada a um timpanograma de pico simples com sonda de alta frequência ${ }^{10-12,16}$

A associação das EOAT com os testes de admitância e reflectância acústica reduz a taxa de falso positivo das EOAT e pode levar a um melhor entendimento das falhas na TAN nos RN com função coclear normal $^{16-21}$. Em um estudo sobre achados timanométricos em 110 neonatos com EOA presente, utilizando tom sonda de $226 \mathrm{~Hz}, 678 \mathrm{~Hz}$ e $1000 \mathrm{~Hz}$, foram encontrados maior ocorrência de curva pico único em $1000 \mathrm{~Hz}$, concluindo que a sonda de $1000 \mathrm{~Hz}$, apresenta, estatisticamente, melhores resultados na caracterização dos timpanogramas em relação à normalidade nesta população ${ }^{17}$. Estudos afirmam que a avaliação da função da orelha média em conjunto com as EOA.
Sabe-se que as EOA são muito sensíveis a efusão de orelha média, sendo possível o aumento da taxa de falha na TAN ocorrer em crianças com diminuição da mobilidade do tímpano, uma vez que a frequência de ressonância da orelha média é mais baixa em lactentes do que em adultos, o que poderia justificar a presença de pico duplo quando se utiliza o tom de frequência timpanométrica em $226 \mathrm{~Hz}^{21-24}$.

Desta forma o presente estudo demonstrou a necessidade de utilização da sonda de $1000 \mathrm{~Hz}$ na imitanciometria para avaliar as condições da orelha média em lactentes com idade inferior a seis meses.

No estudo da ocorrência das EOAT e da timpanometria (Tabela 5) em relação à variável gênero não houve diferença estatisticamente significante, apesar de encontrar maior ocorrência de resultados alterados no sexo feminino, diferindo de alguns estudos realizados que encontraram maior prevalência de EOA ausente no sexo masculino e na OE, com maior amplitude das EOA para o sexo feminino ${ }^{18 ; 25}$. Tais diferenças talvez possam ser atribuídas ao fato da triagem ter sido realizada em equipamento automático que ao atingir o critério interrompe o exame e também pelo tipo de análise que se restringiu a presença ou ausência de respostas e não detalhou o nível da resposta.

\section{CONCLUSÃO}

A timpanometria com sonda de $1000 \mathrm{~Hz}$ teve maior correlação com as EOAT tanto para identificação de respostas em orelhas médias normais como para as alterações de orelha média. Já a timpanometria com sonda de $226 \mathrm{~Hz}$ teve maior correlação apenas nos resultados de orelhas médias normais. Diante destes achados, recomenda-se o uso da timpanometria com tom teste de $1000 \mathrm{~Hz}$ para avaliação da orelha média em lactentes com idade inferior a seis meses. 


\begin{abstract}
Purpose: to compare the findings of TEOAE with tympanometry in 226 and $1000 \mathrm{~Hz}$ in infants. Method: the study was conducted in the Department of Otolaryngology, Hospital Santa Juliana Maceio - Alagoas. We selected infants of both genders, aged from newborn to six months. We excluded those with cleft palate, external and/or middle ear malformation, syndromes, and family history of hearing loss. The following procedures were conducted: anamnesis in order to identify hearing impairment and to select the sample according to the criteria of inclusion and exclusion; otorrinolaringological assessment using otoscopy and hearing evaluation that consisted of transient evoked otoacoustic emissions (TEOAE) and tympanometry with 226 and $1000 \mathrm{~Hz}$ probe tones. In order to analyze the qualitative variables, we used the test for Equality of Two Proportions, and the Chi-Square for Independence and the confidence interval for the average used for the analysis of the results of quantitative variables. Results: comparing the results of TEOAE with the tympanometry results that were obtained with $226 \mathrm{~Hz}$ and $1000 \mathrm{~Hz}$ probe tones, we observed an association of TEOAE with the $1000 \mathrm{~Hz}$ probe for both ears, the infants with TEOAEs absence had altered tympanogram results and the ones where the TEOAEs were present had normal tympanograms. Conclusion: There was a higher correlation between the results of the $1000 \mathrm{~Hz}$ probe tympanometry and the TEOAE in order to identify normal ear responses and middle ear disorders.
\end{abstract}

KEYWORDS: Otoacoustic Emissions, Spontaneous; Acoustic Impedance Tests; Otoscopy

\section{REFERÊNCIAS}

1. Northon JL, Downs MP. Audição na infância. $5^{\text {a }}$ Edição. Guanabara Koogan. 2005. p.63-4. [edição brasileira].

2. Pereira PKS. Ocorrência de alteração de orelha média em recém nascidos que falharam na triagem auditiva neonatal. [Tese]. São Paulo: Universidade Federal de São Paulo; 2009.

3. Akdogan O, Ozkan S. Otoacoustic emissions in children with otitis media with effusion. International Journal of Pediatric Otorhinolaryngology. 2006;70:1941-4.

4. Azevedo MF. Emissões otoacústicas. Figueiredo MS. Emissões otoacústicas e BERA. São José dos Campos: Pulso; 2003. p.53-7.

5. Uchida Y, Ando F, Nakata S, Ueda H, Nakashima $\mathrm{T}$, Niino $\mathrm{N}$, et al. Distortion product otoacoustic emissions and tympanometric measurements in an adult population-based study. Auris Nasus Larynx. 2006;33:397-401.

6. Linares AE, Carvallo RMM. Medidas imitanciométricas em crianças com ausência de emissões otoacústicas. Rev Bras Otorrinolaringol. 2008;74(3):410-6.

7. Saes SO, Goldberg TBL, Montovani JC. Secreção na orelha média em lactentes - ocorrência, recorrência e aspectos relacionados. J Pediatr. 2005;81 (2): 133-8.

8. Doyle KJ, Kong YY, Strobel K, Dallaire $P$, Ray RM. Neonatal middle ear effusion predicts chronic otitis media with effusion. Otol Neurotol. 2004;25(3):318-22.

9. Pereira PKS, Martins A, Vieira MR, Azevedo MF. Programa de triagem auditiva neonatal: associação entre perda auditiva e fatores de risco. Pró-fono. 2007;19(3): 267-78.

10. Ho VMD, Daly KA, Hunter LL, Davey $\mathrm{CM}$. Otoacustic emisson and tympanometry screening among 0-5 year olds. Layngoscope. 2002;112(3):513-9.

11. Margolis RH, Bass-Ringdahl S, Hanks WD, Holte L, Zapala DA. Tympanometry in newborn infants: 1KHz norms. J Am Acad Audiol. 2003;14:383-92.

12. Garcia MV, Azevedo MF, Testa JR. Medidas de imitância acústica em lactentes com $226 \mathrm{~Hz}$ e 1000Hz: correlação com as emissões otoacústicas e o exame otoscópico. Rev Bras otorrinolaringol. 2009;75(1):80-9.

13. Mata J, Rando I, Shepherd J, Miguelez F, Delgado J. Importância de La impedanciometria dentro de um programa de screening auditivo com otoemisiones em ninos. Acta Otorrinolaringol Esp. 2001;52:96-100.

14. Boone RT, Bower CM, Martin PF. Failed Newborn Hearing Screens as presentation for otitis media with effusion in the newborn population. Int $\mathrm{J}$ Pediatr Otorhinolaryngol. 2005;69(3):393-7.

15. Hof JR, Anteunis LJC, Chenault MN, van Dijk P. Otoacoustic emissions at compensated middle ear pressure in children. Int $\mathrm{J}$ Audiol. 2005;44(6):317-20. 
16. Keefe DH, Folsom RC, Gorga MP, Vohr BR, Bulen JC, Norton SJ. Identification of neonatal hearing impairment: ear-canal measurements of acoustic admittance and reflectance in neonates. Ear Hear. 2000;21:443-61.

17. Silva KAL, Novaes BACC, Lewis DR, Carvallo RMM. Achados timpanométricos em neonatos: medidas e interpretações. Rev Bras Otorrinolaringol. 2007;73(5):633-9.Koivunem P, Uhari M, Laitakari K, Alho OP, Luotonem J. Otoacustic emissions and tympanometry in children with otitis media. Ear Hear. 2000;21;212-7.

18. Basseto M, Chiari BM, Azevedo MF. Emissões otoacústicas evocadas transientes (EOAT): amplitude da resposta em recémnascidos a termo e pré-termo. Rev Bras Otorrinolaringol.2003;69(1):84-92.

19. Bochinia CFP, Roggia SM, Cordeiro MLS. Influência do refluxo gastroesofágico na indicação do reteste na triagem auditiva neonatal. Academia Brasileira de Audiologia. Anais de $23^{\circ}$ encontro de Audiologia. Itajaí - SC. 2008. p.1821.
20. Kei J, Allison-Levick J, Dockray J, Harrys R, Kirkegard C, Wong J, et al. High frequency (1000 $\mathrm{Hz}$ ) tympanometry in normal neonates. J Am Acad Audiol. 2003;14:20-8.

21. Doyle KJ, Rodjers P, Fugikawa S, Newman E. External and middle ear effects on infant hearing screening test results. Otolaryngol. Head Neck Surg. 2000;122(4): 477-81.

22. André KD, Sanches SGG, Carvallo RMM. Variação da frequência de ressonância da orelha média em lactentes. 2010 (prelo).

23. Doyle JW, Winther B, Alper C. Daily tympanometry as a functional measure of middle ear status and Eustachian tube function. Auris Nasus Larynx. 2009;36: 20-5.

24. Andrade DHL, Pedruzzi CM, Soares IA, Azevedo MF, Camboim ED. Achados audiológicos e de linguagem em gêmeas regurgitadoras. Rev CEFAC. 2009;11(3):529-38.

25. Dantas MBS, Anjos CAL, Camboim ED, Pimentel MCR. Resultados de um programa de triagem auditiva neonatal em Maceió. Rev. bras. Otorrinolaringol. 2009; 75(1):58-63.
http://dx.doi.org/10.1590/S1516-18462011005000090

RECEBIDO EM: 05/11/2010

ACEITO EM: 05/04/2011

Endereço para correspondência:

Elizângela Dias Camboim

Rua Cel. Alcides de Barros Ferreira, no 26

apt. 301 - Jatiúca

Maceió - AL

CEP: $57036-480$

E-mail: elicamboim@yahoo.com.br 